\title{
Molecular subtypes of small cell lung cancer transformed from adenocarcinoma after EGFR tyrosine kinase inhibitor treatment
}

\author{
Soohyun Hwang ${ }^{1 \#}$, Tae Hee Hong ${ }^{2,3 \#}$, Sehhoon Park ${ }^{4 \#}$, Hyun-Ae Jung ${ }^{4}$, Jong-Mu Sun ${ }^{4}$, Jin Seok Ahn ${ }^{4}$, \\ Myung-Ju Ahn ${ }^{4}$, Keunchil Park ${ }^{4}$, Yoon-La Choi ${ }^{1}$, Se-Hoon Lee ${ }^{4}$ \\ ${ }^{1}$ Department of Pathology and Translational Genomics, Samsung Medical Center, Sungkyunkwan University School of Medicine, Seoul, Republic \\ of Korea; ${ }^{2}$ Department of Thoracic and Cardiovascular Surgery, Samsung Medical Center, Sungkyunkwan University School of Medicine, Seoul, \\ Republic of Korea; ${ }^{3}$ Department of Digital Health, Samsung Advanced Institute for Health Science \& Technology (SAIHST), Sungkyunkwan \\ University, Seoul, Republic of Korea; ${ }^{4}$ Division of Hematology-Oncology, Department of Medicine, Samsung Medical Center, Sungkyunkwan \\ University School of Medicine, Seoul, Republic of Korea \\ Contributions: (I) Conception and design: S Hwang, TH Hong, S Park, YL Choi, SH Lee; (II) Administrative support: S Hwang, TH Hong, S Park; (III) \\ Provision of study materials or patients: S Hwang, TH Hong, S Park, YL Choi, SH Lee; (IV) Collection and assembly of data: All authors; (V) Data \\ analysis and interpretation: S Hwang, TH Hong, S Park; (VI) Manuscript writing: All authors; (VII) Final approval of manuscript: All authors. \\ "These authors contributed equally to this work. \\ Correspondence to: Professor Yoon-La Choi, MD, PhD. Department of Pathology and Translational Genomics, Samsung Medical Center, \\ Sungkyunkwan University School of Medicine, 81 Irwon-ro, Gangnam-gu, Seoul 06351, Republic of Korea. Email: ylachoi@skku.edu; Professor Se- \\ Hoon Lee, MD, PhD. Division of Hematology-Oncology, Department of Medicine, Samsung Medical Center, Sungkyunkwan University School of \\ Medicine, 81 Irwon-ro, Gangnam-gu, Seoul 06351, Republic of Korea. Email: sehoon.lee119@gmail.com.
}

Background: A certain proportion of non-small cell lung cancer (NSCLC) with activating EGFR mutations showed resistance to tyrosine kinase inhibitors (TKIs) by transforming their histology into small cell lung cancer (SCLC). In this study, we evaluated the molecular characteristics of transformed SCLCs.

Methods: Eighteen SCLC tissue samples transformed after EGFR TKI treatment were used for the analysis. Immunohistochemistry was conducted to evaluate the molecular subtype using antibodies representative of the major transcriptional factor-based molecular subtypes, ASCL1 (SCLC-A), NEUROD1 (SCLC-N), POU2F3 (SCLC-P), and YAP1. Subtypes were categorized based on a predefined criteria.

Results: Among the study population $(n=18)$, most of the patients were initially diagnosed with adenocarcinoma ( $n=17)$, and one patient was diagnosed with adenosquamous histology. Eight patients (44.4\%) were never-smokers, and nine patients were women (50.0\%). Staining of pre-transformation sample was conducted in six patients, and five of them showed no discernible expression for ASCL1, NEUROD1, or POU2F3. However, the proportion of molecular subtypes after SCLC transformation was predominantly SCLC-N (n=9, 50.0\%), followed by SCLC-Triple Negative (SCLC-TN; n=5, 27.8\%) and SCLC-A ( $\mathrm{n}=4$, 22.2\%). The median overall survival from TKI initiation was longer in patients who transformed to SCLC-A $(\mathrm{P}=0.009)$ than in those who transformed to either SCLC-N or SCLC-TN. However, the overall survival difference since SCLC transformation was not significant $(\mathrm{P}=0.370)$.

Conclusions: In our series, SCLC-N subtype was prevalent in SCLC transformed after EGFR TKI treatment. In addition, overall survival and the time to SCLC transformation from the EGFR TKI treatment were longer in patients who transformed to the SCLC-A type. Large-scale data will be required to confirm our findings.

Keywords: Histologic transformation; small cell lung cancer (SCLC); molecular subtype; epidermal growth factor receptor; tyrosine kinase inhibitor (TKI)

Submitted Aug 30, 2021. Accepted for publication Oct 09, 2021.

doi: $10.21037 /$ tlcr-21-691

View this article at: https://dx.doi.org/10.21037/tlcr-21-691 


\section{Introduction}

The primary treatment option for non-small cell lung cancer (NSCLC) adenocarcinoma (ADC) with activating epidermal growth factor receptor (EGFR) mutation is EGFR tyrosine kinase inhibitor (TKI) (1). After a certain period of treatment with EGFR TKI, acquired resistance emerges most frequently with a secondary mutation, p.T790M (36-50\%), followed by MET amplification (10-19\%) (2). Interestingly, up to $3-5 \%$ of patients experience histological transformation into small cell lung cancer (SCLC). As an underlying mechanism, ADC with a predisposed clonally inactivated $\mathrm{Rb}$ and $\mathrm{p} 53$ mutation and APOBEC mutation signature is known to be associated with SCLC transformation (3).

The transformed SCLC harbors similar morphological and immunohistochemical (IHC) characteristics as those observed in de novo SCLC, including high expression of chromogranin and synaptophysin (4). However, little is known about the clinical outcomes of transformed SCLC, with limited studies arguing that their outcomes are similar to those of de novo SCLC, where the median overall survival is approximately 9 to 10 months after the transformation $(5,6)$. In addition, the time-course of transformation and molecular characteristics of transformed SCLC is not well known.

For several decades, de novo SCLC has been considered as a single disease entity despite previous evidence showing a different SCLC subset with distinct morphology and gene expression profile, which may be associated with poor clinical outcomes (7). From a genomic perspective, the tumor was once characterized by near-universal damaging alteration of RB1 and TP53. Recently, molecular subtypes of SCLC have been proposed by Rudin et al. based on the relative expression profile of four key transcriptional regulators (ASCL1, NEUROD1, POU2F3, and YAP1) (8). This concept of molecular subtyping inspired many researchers to stratify patients into each subset with distinct therapeutic vulnerabilities. It has also been suggested that subtype plasticity might occur during the course of treatment or natural history and may be partly responsible for therapeutic failure $(9,10)$. Therefore, molecular subtyping of SCLC may contribute to a better understanding of treatment resistance in terms of molecular profiles and improving therapeutic response in a more personalized manner. Nevertheless, there are limited data linking molecular subtypes of transformed SCLC after EGFR-TKI treatment and their clinical outcomes.
In light of the potential significance of molecular subtypes and paucity of management guidelines in transformed SCLC, we aimed to investigate the molecular subtypes associated with transformation and their impacts on clinical outcomes. Our study provides a basic resource for developing novel diagnostic and therapeutic approaches by leveraging subtype information of transformed SCLC cases. We present the following article in accordance with the STROBE reporting checklist (available at https://dx.doi. org/10.21037/tlcr-21-691).

\section{Methods}

\section{Study design and materials}

From January 2009 to December 2020, patients with EGFR-mutated SCLC and with an initial histology of NSCLC were included in this study. We excluded patients with unavailable tissue, poor specimen quality, or patients with no previous TKI treatment. In addition, patients with de novo combined SCLC (e.g., NSCLC coexisting with the SCLC component at the initial diagnosis) were excluded. Collected data included demographic information, pre- and post-transformation clinical data on tumor histology and molecular pathology, the type and duration of systemic therapeutics used, and survival. A consort diagram of the patient inclusion is shown in Figure 1 . The study was approved by the Samsung Medical Center Institutional Review Board (SMC 2021-07-060001), and the need for patient consent was waived because of the retrospective nature of this study. This study was performed in accordance with the Declaration of Helsinki (as revised in 2013).

\section{Immunobistochemistry antibody and interpretation}

IHC staining was conducted on $4 \mu \mathrm{m}$-thick formalin-fixed paraffin-embedded (FFPE) tissue sections, using primary antibodies for ASCL1 (Clone 4B72D11.1, Cat\# 556604, BD Biosciences, 1:25), NEUROD1 (Clone EPR20766, Cat\# ab213725, Abcam, 1:250), POU2F3 (polyclonal, Cat\# NBP1-83966, Novus Biologicals: 1:200), YAP1 (Clone 2F12, Cat\# ab56701, Abcam, 1:200), RB1 (Clone 4H1, Cat\# 9309, Cell Signaling Technologies, 1:100), and p53 (clone DO-7, Cat\#NCL-L-p53-DO7, Leica Biosystems, 1:400). RB1 status was categorized as loss (no nuclear staining) or intact (retained nuclear staining). The result of p53 IHC was interpreted as complete absence (no nuclear staining), 
Consort diagram and study purposes

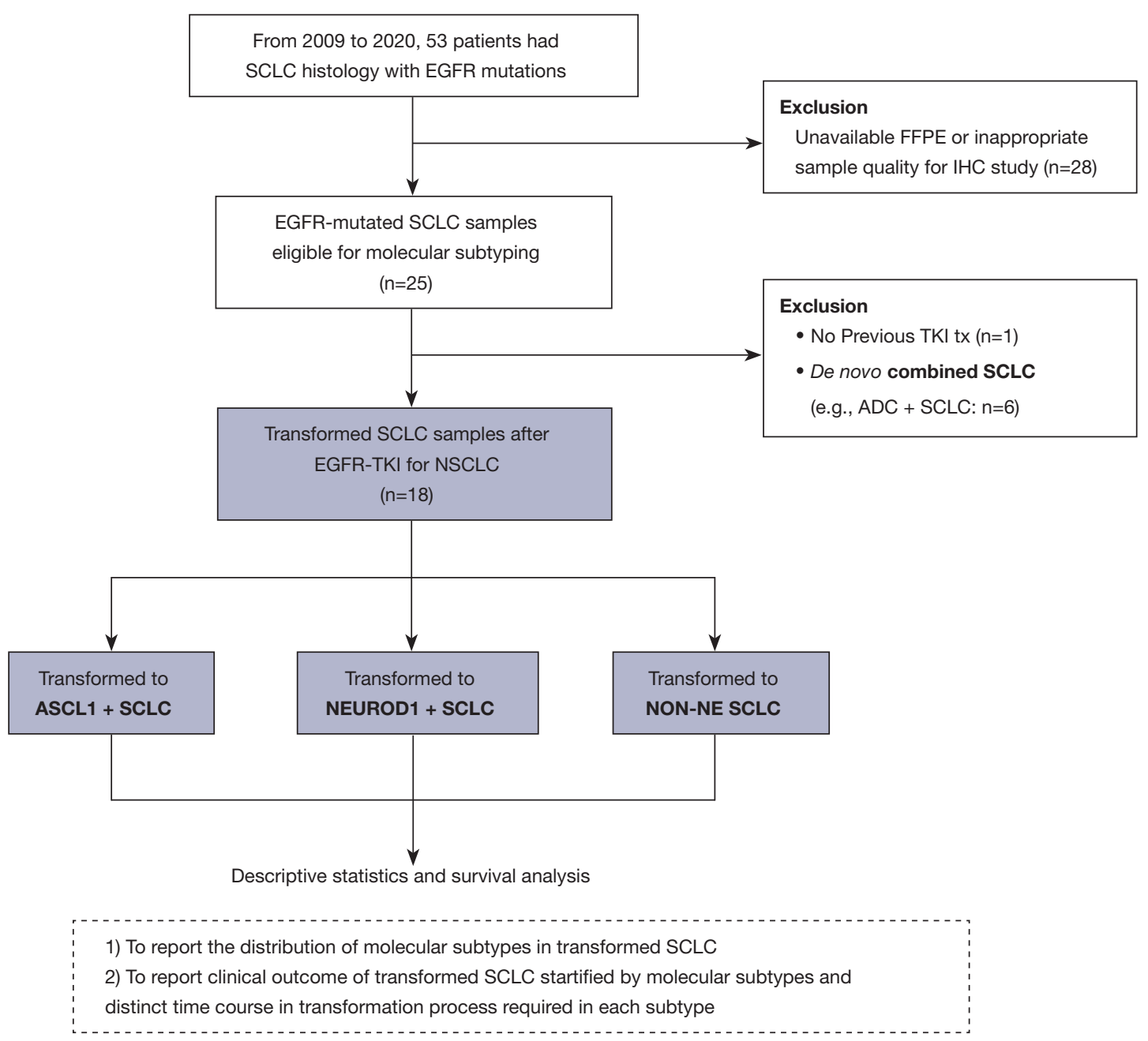

Figure 1 Consort diagram for the study population.

overexpression (strong intensity in $70 \%$ or more of the tumor cell nuclei) or wild type (variable intensity in less than $70 \%$ of the tumor cell nuclei) (11). As different intensities of the positive cells were observed, the intensity ( 0 to $3+)$ and proportion (\%) of the positive cells were recorded. $\mathrm{H}$-score was derived as the sum of the proportion multiplied by its corresponding intensity (ranging from 0 to 300) based on a previous study (12). An H-score of more than 50 was considered significant. Based on the H-score, samples were classified as either SCLC-A, ASLC1 expression dominant, SCLC-N, NEUROD1 expression dominant, and SCLCTN (triple-negative). Subtype evaluation was conducted by two independent pathologists who had no access to clinical outcomes.
In addition, an exploratory IHC analysis was conducted on EGFR-mutated, de novo combined SCLC samples ( $\mathrm{n}=7$ ) to identify the spatial differences in staining patterns in each area of NSCLC and SCLC.

\section{Definition of outcomes and statistical analysis}

The primary purpose of this study was to evaluate the prevalence of each molecular subtype in transformed SCLC after TKI treatment. As exploratory outcomes, transformation-free survival and overall survival were evaluated.

Overall survival was defined as the time between the date of initiation of TKI treatment to that of death or the last 
follow-up for censored patients. Time-to-transformation was defined as the time between the date of initiation of TKI treatment and that of re-biopsy demonstrating the transformation into SCLC histology. Post-transform survival was defined as the time between the date of rebiopsy demonstrating SCLC to death or the last follow-up for censored patients.

For non-normally distributed continuous variables, median and interquartile range values were reported. Categorical variables were reported as numbers with percentages and were compared using Fisher's exact test.

Survival curves for overall survival, transformationfree survival, and post-transformed survival were estimated using the Kaplan-Meier method and compared between groups using log-rank statistics. Cox proportional hazard regression was used to examine the adjusted effects of the molecular subtypes in the multivariable model. All statistical analyses were conducted using $\mathrm{R}$ software (R Foundation for Statistical Computing, Vienna, Austria, version 3.6.1).

\section{Results}

\section{Baseline demographics}

A total of 53 patients with a history of EGFR-mutated SCLC were identified. After applying predefined exclusion criteria, the final cohort consisted of 18 TKI-treated patients with adequate specimen quality available for SCLC molecular subtyping (Figure 1).

The cohort included nine female and nine male patients with a median age at diagnosis of 55 years. The racial makeup was $100 \%$ Asian, and $44.4 \%$ of these had never smoked (Figure $2 A$ ). The patients had pre-transformation histology of adenocarcinoma ( $\mathrm{n}=17,94.4 \%)$ or adenosquamous carcinoma $(\mathrm{n}=1,5.6 \%)$. All patients had EGFR activating mutations, including $13(72.2 \%)$ with exon 19 deletion mutations and $5(27.8 \%)$ with exon 21 p.L858R mutations.

All patients received at least one line of EGFRTKI therapy. Seven patients (39\%) received secondline EGFR TKI treatment based on the acquired EGFR p.T790M mutation before being identified for the SCLC transformation. Only one patient received third-generation TKI (osimertinib) as first-line therapy.

\section{Characteristics of transformed SCLC and molecular subtype distribution}

All transformed histologies were confirmed to be
SCLC. The founder EGFR mutation was conserved in all transformed cases, as confirmed by the Cobas EGFR mutation test (Roche Diagnostic). The time to transformation is depicted in Figure $2 B$ (thick line) in decreasing order. The median time to transformation after initiating TKI therapy was 18.3 months (95\% CI, 12.4 to 24.4 months).

The most common molecular subtype after SCLC transformation was SCLC-N, which comprised half of the total cases $(n=9)$. SCLC-TN accounted for 5 cases (27.8\%), and SCLC-A type was identified in 4 cases (22.2\%). Otherwise, POU2F3 and YAP1 were weakly expressed. Loss of expression of RB1 was observed in 14 cases $(82.4 \%$ of examined cases), and p53 was abnormally expressed in 15 cases (overexpression, $\mathrm{n}=9$; complete absence, $\mathrm{n}=6$ ). TTF1 staining was maintained in 16 cases $(88.9 \%)$ (Table S1). Representative images with clinical information are shown in Figure 3.

To identify the dominance of SCLC-N as a unique pattern in transformed SCLC, the subtype distribution in our cohort was compared with that of two representative cohorts of de novo SCLC containing subtype information (Table 1) $(8,13)$. We observed discernible enrichment of SCLC-N type in the TKI-treated cohort (Fisher's exact test, $\mathrm{P}<0.001)$. In contrast, the SCLC-A type was more prevalent in public cohorts of de novo SCLC (Fisher's exact test, $\mathrm{P}<0.001$ ).

\section{Clinical outcomes}

The median overall survival was 33.7 months [95\% confidential interval (CI), 32.8-36.0] after initiating TKI treatment and the median transformation-free survival was 18.3 months (95\% CI, 12.4-24.4 months) (Figure 4A,4B). Interestingly, we observed intergroup differences between the molecular subtypes $(\log$-rank $\mathrm{P}=0.009)$. Patients who transformed to SCLC-A showed the longest overall survival after TKI treatment compared to the overall survival of SCLC-N or SCLC-TN patients. The median overall survival for each group was 47.6 months (95\% CI, 35.6-55.2 months) for SCLC-A, 33.7 months (95\% CI, 32.8-37.3 months) for SCLC-N, and 24.6 months (95\% CI, 24.0-26.1 months) for SCLC-TN. The median overall survival after SCLC transformation was 17.1 months (95\% CI, 12.6-22.0 months) (Figure 4C). However, we did not observe any significant differences in post-transform survival (log-rank $\mathrm{P}=0.370$ ).

Multivariable logistic regression revealed that the molecular subtype of transformed SCLC was an 
A
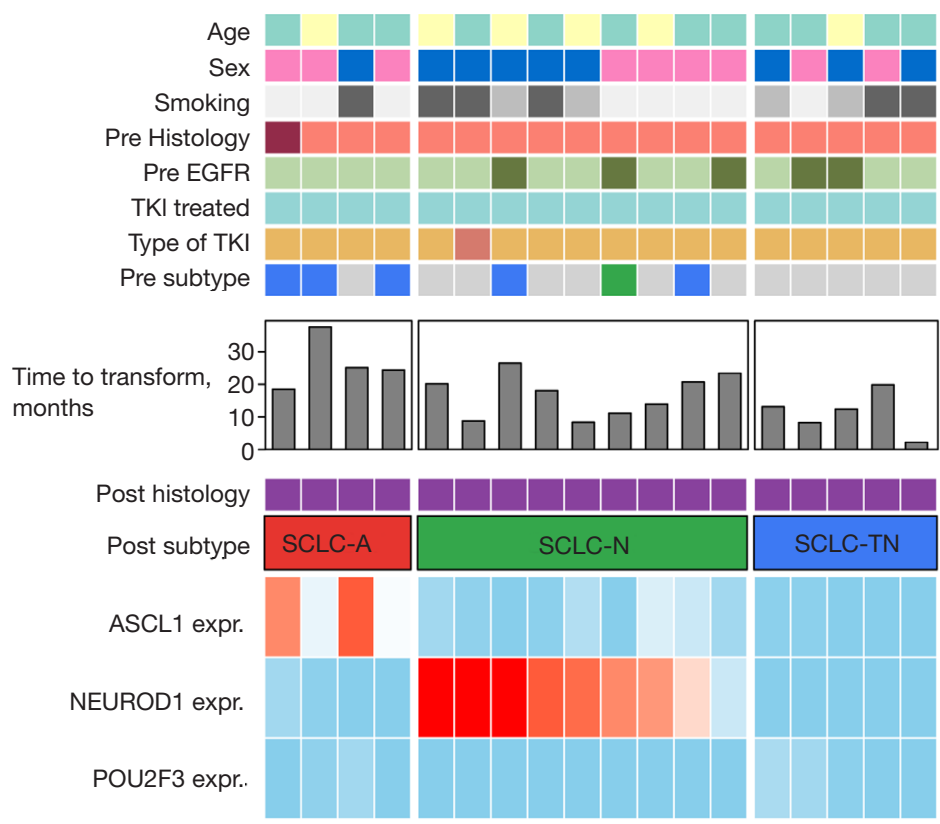

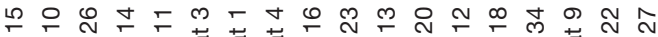

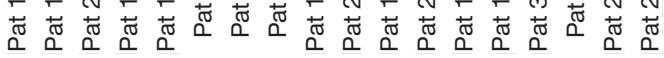

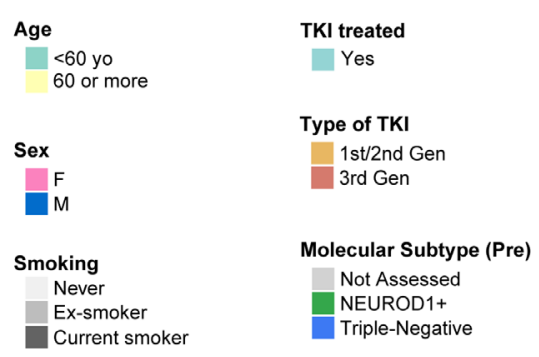

Pretransform histology LUAD

Adenosquamous

Pretransform EGFR status

Exon 19 Del

Exon 21 L858R
Molecular Subtype (Post) SCLC-A SCLC-N

Post-transform histology $\square$ SCLC

B

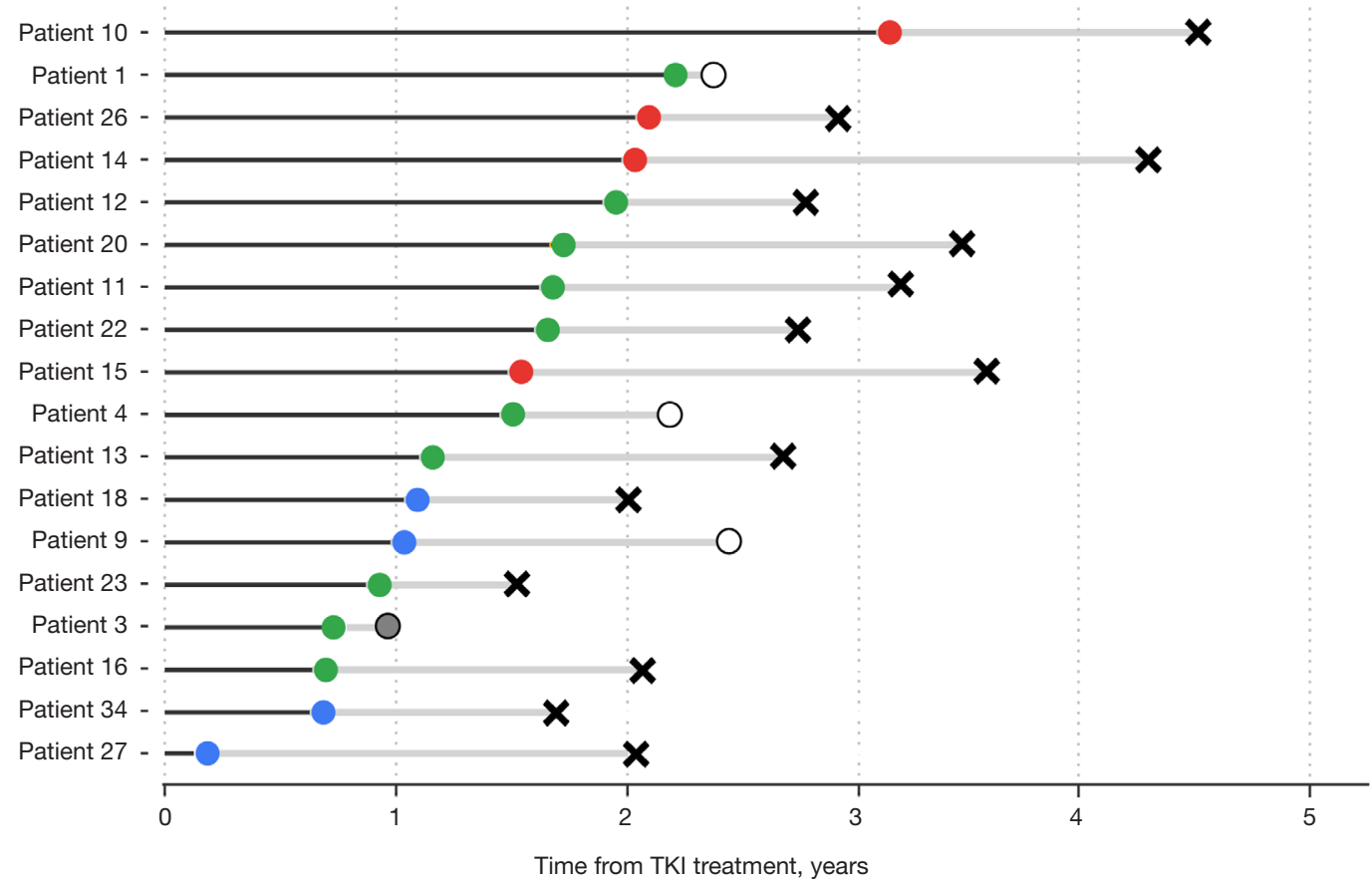

Figure 2 Clinicopathologic characteristics of the cohort. (A) Overall baseline demographics and expression profile of ASCL1, NEUROD1, and POU2F3. (B) Swimlane plot for time to transformation and post transformed survival. EGFR, epidermal growth factor receptor; TKI, tyrosine kinase inhibitor; SCLC, small cell lung cancer. 


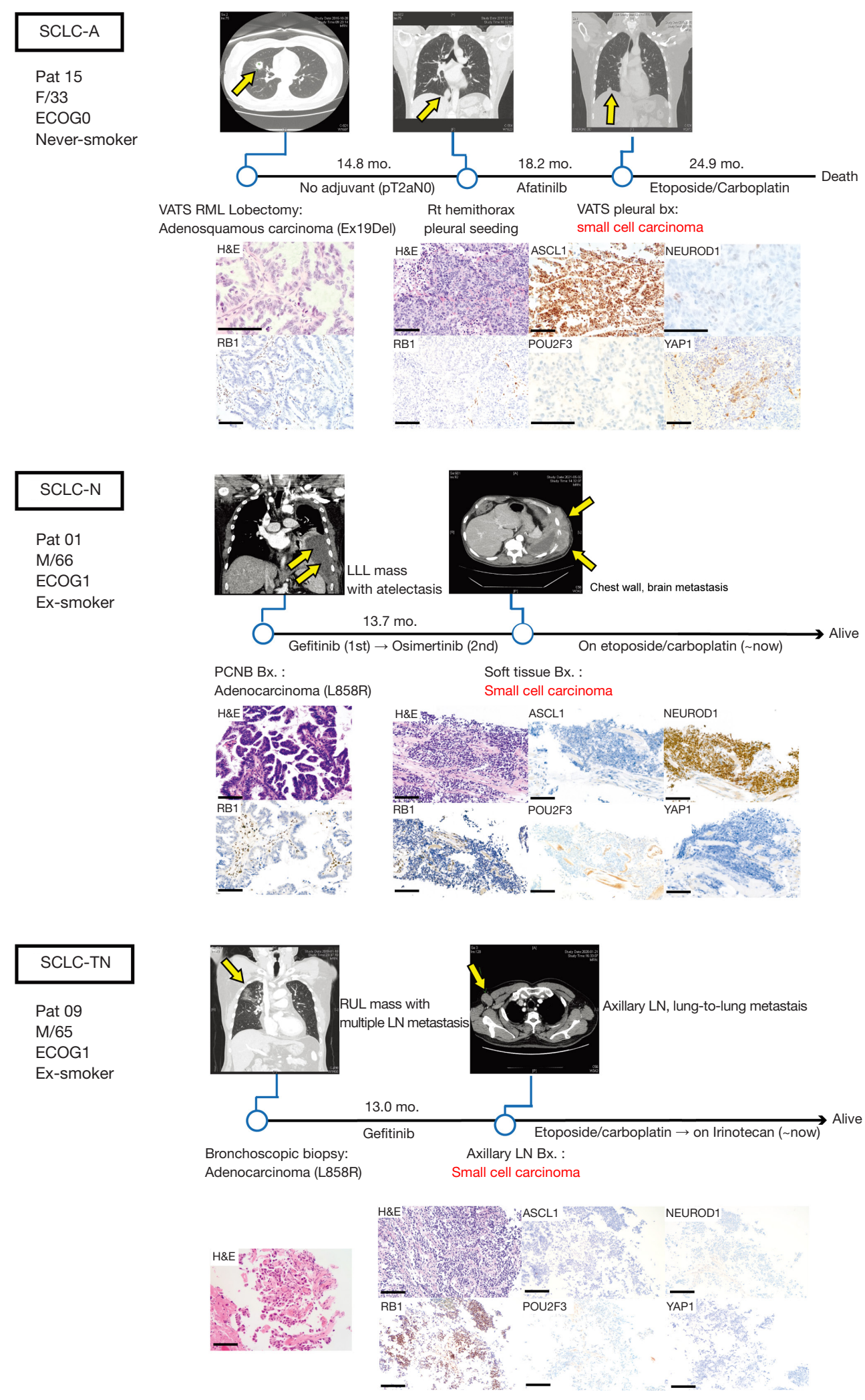

Figure 3 Representative cases for SCLC-A, SCLC-N, and SCLC-TN with immunohistochemistry results and clinical outcomes (hematoxylin-eosin, RB1, ASCL1, NEUROD1, POU3F3 and YAP1 stain; scale bars =100 $\mu$ ). Arrows indicate the region of disease progression. SCLC, small cell lung cancer. 
Table 1 Molecular subtype distribution of SCLC: transformed SCLC vs. de novo SCLC cohorts

\begin{tabular}{|c|c|c|c|c|c|}
\hline Subtypes & $\begin{array}{c}\text { This study } \\
\mathrm{N}(\%)\end{array}$ & \multicolumn{2}{|c|}{ Rudin et al. (2019) ${ }^{\dagger}$} & \multicolumn{2}{|c|}{ Baine et al. (2020) } \\
\hline Total & $18(100.0)$ & $81(100.0)$ & $8.1 \mathrm{e}-08$ & $159(100.0)$ & $9.3 e-05$ \\
\hline \multicolumn{6}{|l|}{ NE } \\
\hline SCLC-A & $4(22.2)$ & 57 (70.3) & $<0.001$ & $110(69.2)$ & $<0.001$ \\
\hline \multicolumn{6}{|l|}{ Non-NE } \\
\hline SCLC-P & $0(0.0)$ & $13(16.0)$ & $<0.001$ & $10(6.3)$ & $<0.001$ \\
\hline SCLC-Y & $0(0.0)$ & $2(2.5)$ & NS & $0(0.0)$ & NS \\
\hline SCLC-all negative & $5(27.8)$ & $0(0.0)$ & $<0.001$ & $12(7.5)$ & $<0.001$ \\
\hline
\end{tabular}

${ }^{\dagger}$, cell line data were removed for proportion analysis; *, Fisher's exact test. SCLC, small cell lung cancer; NE, neuroendocrine; NS, not significant.

independent prognostic factor for overall survival and transformation-free survival (Figure $5 A, 5 B$ ). Among the subtypes, SCLC-TN seems to be associated with a poorer prognosis (adjusted HR for overall survival 56.27, 95\% CI, $2.5-1,300, \mathrm{P}=0.012)$. Similarly, SCLC-N was also associated with a shorter transformation-free survival as compared to SCLC-A (adjusted HR for transformation-free survival 7.94, $95 \%$ CI, $1.30-47.9, \mathrm{P}=0.024)$.

\section{Exploratory analysis of de novo combined SCLC and pre- transformation samples}

We conducted exploratory IHC analysis in de novo combined SCLC samples $(\mathrm{n}=7)$, which comprised 4 combined SCLC/ADC, 2 combined SCLC/Large Cell Neuroendocrine Carcinoma (LCNEC), and 1 combined SCLC/ADC/LCNEC (Table S1). Overall, the most common subtype was SCLC-N $(n=3)$, followed by SCLC-A $(n=2)$, SCLC-TN $(n=1)$, and undetermined (mixed expression of ASCL1 and POU2F3, n=1). In all cases, expression of subtype IHC markers was restricted to neuroendocrine components (SCLC/LCNEC) (Figure S1).

In the six cases available for the pre-transformation samples, five showed no discernible staining for ASCL1, NEUROD1, or POU2F3. In these cases, four cases were highly expressed in YAP1, which was not observed in the transformed sample. A single case was highly stained with NEUROD1 only in the focal neuroendocrine component. Complete absence of p53 was identified in 2 cases and overexpression was identified in 4 cases. All six cases showed loss of RB1 expression and were transformed to either SCLC-A (n=3) or SCLC-N ( $=3)$ after the TKI treatment.

\section{Discussion}

In this study, we evaluated molecular subtypes and prognosis of transformed SCLC after EGFR TKI treatment. Despite the limited number of patients and matched pre-treatment samples, several findings of this study should be highlighted.

From this study, we identified that baseline characteristics and clinical behavior of transformed SCLC are different from those of de novo SCLC. In terms of demographics, our population consisted of $50 \%$ female patients and $44.4 \%$ never-smokers. The median overall survival after SCLC transformation was approximately 17.1 months, which is longer than the recently updated overall survival (12 to 13 months) for patients with extensive disease SCLC who treated with front-line immune checkpoint inhibitor $(14,15)$. Moreover, overall survival after TKI treatment was approximately 33.7 months, which was similar to the general population with EGFR-harboring NSCLC patients (16). This might be due to the fact that the behavior of transformed SCLC is more similar to the prognosis of NSCLC rather than SCLC.

It was found that SCLC-A is the predominant subtype of the de novo SCLC, which comprised up to $69 \%$ of the patients, followed by SCLC-N $(20-30 \%)(8,13)$. In a subgroup analysis of our study, the SCLC-N subtype was observed in $50 \%$ of our study population, which was a comparably higher incidence of SCLC-N compared 
A Overall survival
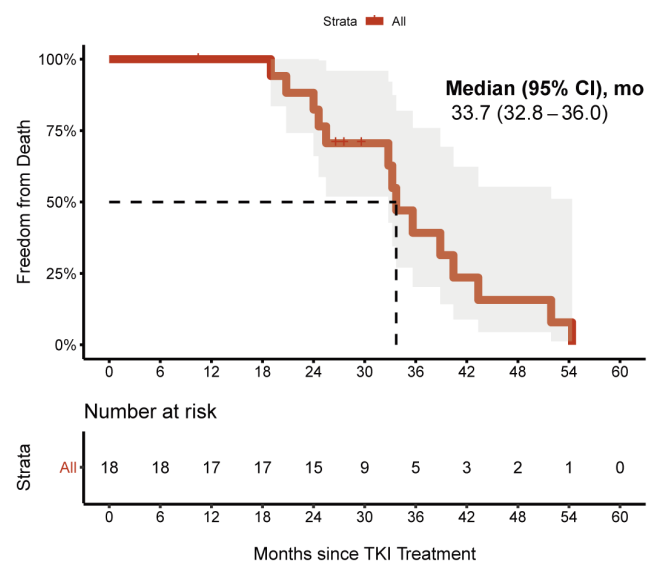

B Transformation-free survival
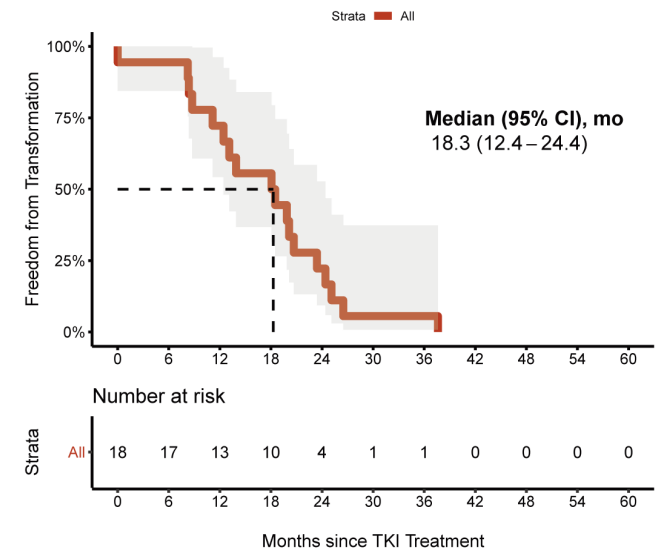

C Post-transform survival
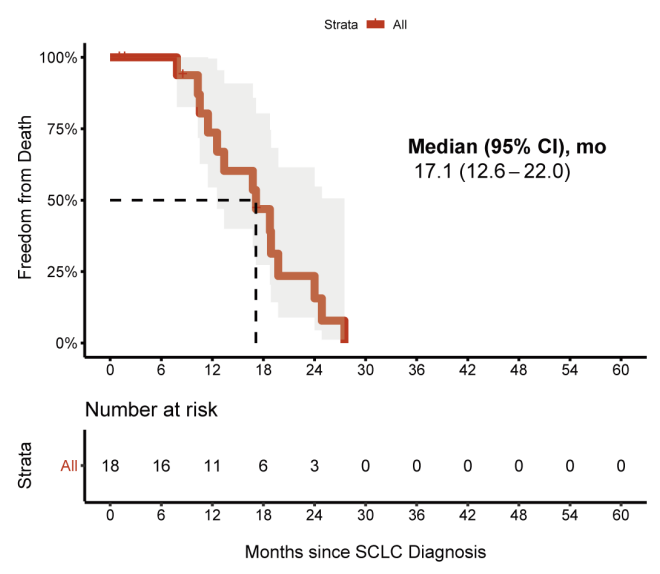
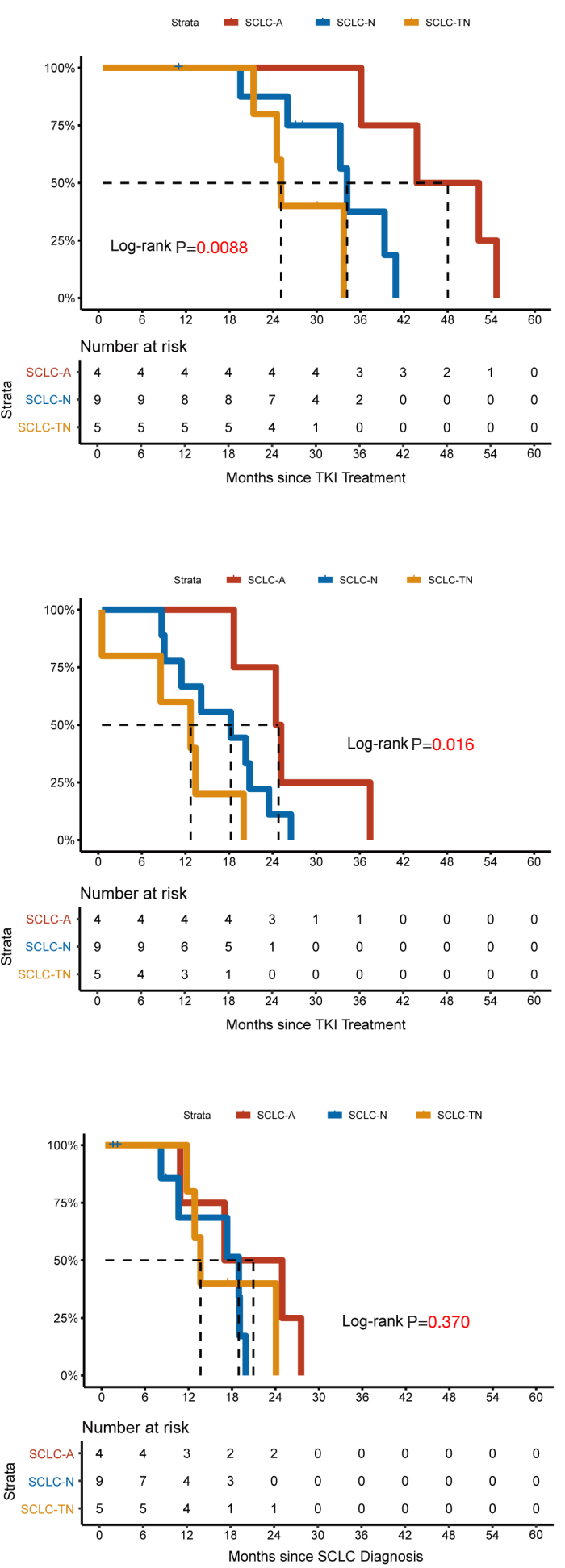

Figure 4 Survival analysis of entire cohort and its stratification according to each molecular subtype. (A) Overall survival from the initiation of EGFR TKI treatment. (B) Transformation-free survival from the initiation of EGFR TKI to the date of histological confirmation of SCLC. (C) The overall survival from the date of histological confirmation of SCLC. EGFR, epidermal growth factor receptor; TKI, tyrosine kinase inhibitor; SCLC, small cell lung cancer. 
A Multivariable Cox regression for overall survival

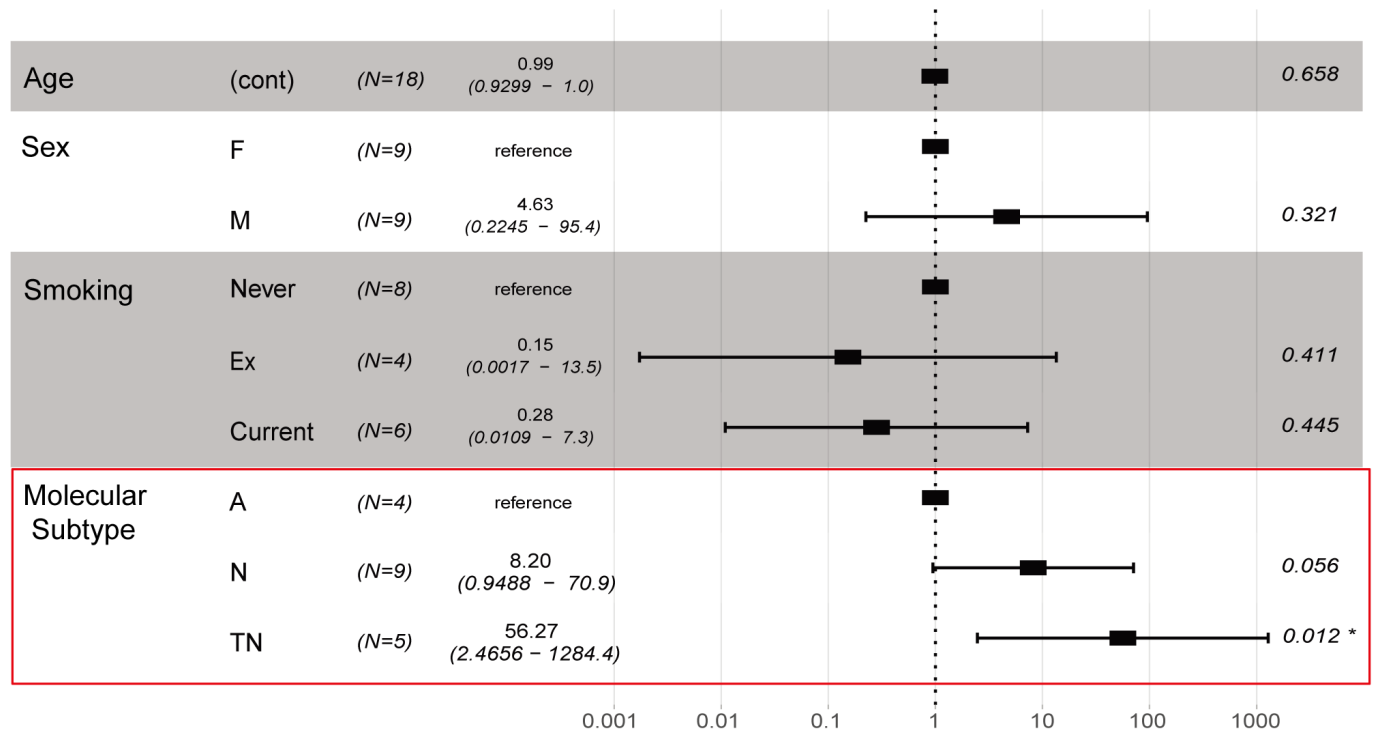

\#Events: 14; Global P value (Log-Rank): 0.078494 AIC: 53.3; Concordance Index: 0.75

B Multivariable Cox regression for transformation-free survival



\#Events: 18; Global P value (Log-Rank): 0.01054 AIC: 53.3; Concordance Index: 0.76

Figure 5 Multivariate cox regression for (A) overall survival and (B) transformation-free survival. ${ }^{*} \mathrm{P}<0.05 ;{ }^{* *} \mathrm{P}<0.01$. 
to the de novo SCLC. A similar finding was observed in a recent report with EGFR-mutated SCLC, where 9 out of 13 patients $(69.2 \%)$ were identified as SCLC-N (17). The references used to compare the incidence of subtypes are from clinical data mostly in North America or Europe. It is known that ethnicity is associated with molecular characteristics of tumor (e.g., high prevalence of EGFRmutant NSCLC in Asia). Therefore, it should be considered in future studies on this topic.

It is known that the SCLC-N subtype is expected to be related to the more aggressive behavior by showing comparably low neuroendocrine differentiation, variant morphology, and activated notch signal promoted by upregulation of MYC (10). From the subgroup analysis, the overall survival of patients who transformed to SCLC-N was shorter (HR 8.20, $\mathrm{P}=0.056$ ) as compared to the SCLC-A subtype (Figure $5 A$ ). In addition, time-totransformation to the SCLC with the EGFR TKI treatment was also shorter in patients who transformed to SCLC-N (18.1 months) as compared to those who transformed to SCLC-A (24.8 months, HR =7.94, $\mathrm{P}=0.024$ ) (Figure 4B). This finding was predominant in SCLC-TN (12.4 months, $\mathrm{HR}=209.34, \mathrm{P}=0.002)$. However, post-transformed survival did not differ between the groups $(\mathrm{P}=0.370)$ (Figure $4 C$ ). Once again, this suggests that the overall prognosis for patients who will potentially experience histological transformation after EGFR TKI is determined by the time to transformation and the transformed molecular subtypes.

Notable findings were observed from histological examination. Although all transformed cases were uniformly diagnosed as SCLC, SCLC-A in our study often showed a mixture of larger cells with more than minimal cytoplasm, as representatively shown in Figure 3. In contrast, these features imparting a transitional NSCLClike morphology were rarely observed in SCLC-N. Additionally, YAP1, which is constantly overexpressed in NSCLC, was observed in all four SCLC-A cases but only in one SCLC-N case (Table S1) (18). These morphological and immunohistochemical findings suggest that SCLC-A, rather than SCLC-N, may retain the biologic characteristics of ADC from which it is derived. In addition, our transformed SCLC sample was predominantly stained by single subtype marker, unlike $38 \%$ of de novo SCLC that were composed of dual expression of ASCL1 and NEUROD1 (13). Similarly, combined SCLC/NSCLC sample in our exploratory analysis were expressed with either of ASCL1 or NEUROD1 (Table S1). This finding is not fully explained by our conventional knowledge that molecular subtypes are interchangeable (10). A recent study using preclinical SCLC model had suggested that molecular subtypes are understood as a spectrum phenomenon, changed by activation of certain transcriptional factors or signaling pathways such as MYC or Notch. More specifically, plasticity seems to have a tendency to follow the order ASCL1 > NEUROD1 > YAP1 (9). Since the majority of our samples expressed a single subtype marker, it is of question that molecular subtypes in transformed SCLC follow the suggested evolution theory in de novo SCLC. We surmise that TKI therapy may induce selective pressure toward switching to a specific subtype when compared to natural evolution occurring without TKI therapy.

There are some limitations to our study. First, our analysis was based on a small number of patients from a biopsy sample in a retrospective manner. Hence, a potentially unexpected bias could be included in the analysis. Second, despite the fact that the median time to the transformation in our study (18.3 months) was very similar to previous reports, post-transformed survival was longer in our study population $(5,6)$. Finally, it was difficult to predict the destiny of the transformation subtype before the EGFR TKI treatment. The exploratory analysis showed that pre-EGFR TKI treatment samples had no unique pattern in the IHC staining of ASCL1, NEUROD1, or POU2F3 but showed a high expression with YAP1.

In conclusion, we identified that the transformed SCLC has different molecular subtypes and histological characteristics compared to de novo SCLC. In addition, transformed molecular subtypes might impact the clinical course of time-to-transformation and overall survival of those who potentially experience SCLC transformation after EGFR TKI treatment. Since this study suffers from the small size of samples, larger data will be required to confirm findings from our series. Based on our preliminary results, the underlying mechanisms that drive and impact the subtype differentiation during SCLC transformation should be elucidated in future studies.

\section{Acknowledgments}

Funding: This work was supported by the National Research Foundation of Korea (NRF) grant funded by the Korean government (MSIT) (2020R1C1C1010626 to Sehhoon Park), the National Research Foundation of Korea (NRF) grant funded by the Korea government (MSIT) (2020R1A2C3006535 to Se-Hoon Lee), the National Cancer Center Grant (NCC1911269-3 to Se-Hoon Lee), 
the grant of the Korea Health Technology R\&D Project through the Korea Health Industry Development Institute (KHIDI), funded by the Ministry of Health \& Welfare, Republic of Korea (HR20C0025 to Se-Hoon Lee), the Samsung Medical Center Grant (SMO120004 to Sehhoon Park), and the 7th AstraZeneca-KHIDI oncology research program (to Sehhoon Park).

\section{Footnote}

Reporting Checklist: The authors have completed the STROBE reporting checklist. Available at https://dx.doi. org/10.21037/tlcr-21-691

Data Sharing Statement: Available at https://dx.doi. org/10.21037/tlcr-21-691

Conflicts of Interest: All authors have completed the ICMJE uniform disclosure form (available at https://dx.doi. org/10.21037/tlcr-21-691). JSA reports personal fees from Amgen, personal fees from Pfizer, personal fees from AstraZeneca, personal fees from Menarini, personal fees from Roche, personal fees from Eisai, personal fees from Boehringer Ingelheim, personal fees from BMSOno, personal fees from MSD, personal fees from Janssen, personal fees from Samsung Bioepis, outside the submitted work. SHL reports grants and personal fees from MSD, personal fees from Novartis, personal fees from AstraZeneca, personal fees from BMS, personal fees from Roche, outside the submitted work. KP reports personal fees from Astellas, Astra Zeneca, AMGEN, Boehringer Ingelheim, Clovis, Eli lilly, Hanmi, KHK, Merck, MSD, Novartis, ONO, Roche, BluePrint, outside the submitted work. SP reports stock holding of Lunit Inc. The other authors have no conflicts of interest to declare.

Ethical Statement: The authors are accountable for all aspects of the work in ensuring that questions related to the accuracy or integrity of any part of the work are appropriately investigated and resolved. This study was performed in accordance with the Declaration of Helsinki (as revised in 2013). This study was reviewed and approved by the Institutional Review Board (SMC 2021-07-060-001) at Samsung Medical Center and individual consent for this retrospective analysis was waived.

Open Access Statement: This is an Open Access article distributed in accordance with the Creative Commons
Attribution-NonCommercial-NoDerivs 4.0 International License (CC BY-NC-ND 4.0), which permits the noncommercial replication and distribution of the article with the strict proviso that no changes or edits are made and the original work is properly cited (including links to both the formal publication through the relevant DOI and the license). See: https://creativecommons.org/licenses/by-nc-nd/4.0/.

\section{References}

1. NCCN Clinical Practice Guidelines in Oncology. NonSmall Cell Lung Cancer. Version 5.2021. Available online: https://www.nccn.org/professionals/physician_gls/default. aspx (accessed Aug 10, 2021).

2. Ke EE, Zhou Q, Zhang QY, et al. A Higher Proportion of the EGFR T790M Mutation May Contribute to the Better Survival of Patients with Exon 19 Deletions Compared with Those with L858R. J Thorac Oncol 2017;12:1368-75.

3. Lee JK, Lee J, Kim S, et al. Clonal History and Genetic Predictors of Transformation Into Small-Cell Carcinomas From Lung Adenocarcinomas. J Clin Oncol 2017;35:3065-74.

4. Oser MG, Niederst MJ, Sequist LV, et al. Transformation from non-small-cell lung cancer to small-cell lung cancer: molecular drivers and cells of origin. Lancet Oncol 2015;16:e165-72.

5. Marcoux N, Gettinger SN, O'Kane G, et al. EGFRMutant Adenocarcinomas That Transform to Small-Cell Lung Cancer and Other Neuroendocrine Carcinomas: Clinical Outcomes. J Clin Oncol 2019;37:278-85.

6. Ferrer L, Giaj Levra M, Brevet M, et al. A Brief Report of Transformation From NSCLC to SCLC: Molecular and Therapeutic Characteristics. J Thorac Oncol 2019;14:130-4.

7. Gazdar AF, Carney DN, Nau MM, et al. Characterization of variant subclasses of cell lines derived from small cell lung cancer having distinctive biochemical, morphological, and growth properties. Cancer Res 1985;45:2924-30.

8. Rudin CM, Poirier JT, Byers LA, et al. Molecular subtypes of small cell lung cancer: a synthesis of human and mouse model data. Nat Rev Cancer 2019;19:289-97.

9. Ireland AS, Micinski AM, Kastner DW, et al. MYC Drives Temporal Evolution of Small Cell Lung Cancer Subtypes by Reprogramming Neuroendocrine Fate. Cancer Cell 2020;38:60-78.e12.

10. Mollaoglu G, Guthrie MR, Böhm S, et al. MYC Drives Progression of Small Cell Lung Cancer to a Variant 
Neuroendocrine Subtype with Vulnerability to Aurora Kinase Inhibition. Cancer Cell 2017;31:270-85.

11. Köbel M, Piskorz AM, Lee S, et al. Optimized p53 immunohistochemistry is an accurate predictor of TP53 mutation in ovarian carcinoma. J Pathol Clin Res 2016;2:247-58.

12. Flowers JL, Burton GV, Cox EB, et al. Use of monoclonal antiestrogen receptor antibody to evaluate estrogen receptor content in fine needle aspiration breast biopsies. Ann Surg 1986;203:250-4.

13. Baine MK, Hsieh MS, Lai WV, et al. SCLC Subtypes Defined by ASCL1, NEUROD1, POU2F3, and YAP1: A Comprehensive Immunohistochemical and Histopathologic Characterization. J Thorac Oncol 2020;15:1823-35.

14. Goldman JW, Dvorkin M, Chen Y, et al. Durvalumab, with or without tremelimumab, plus platinum-etoposide versus platinum-etoposide alone in first-line treatment

Cite this article as: Hwang S, Hong TH, Park S, Jung HA, Sun JM, Ahn JS, Ahn MJ, Park K, Choi YL, Lee SH. Molecular subtypes of small cell lung cancer transformed from adenocarcinoma after EGFR tyrosine kinase inhibitor treatment. Transl Lung Cancer Res 2021;10(11):4209-4220. doi: 10.21037/ tlcr-21-691 of extensive-stage small-cell lung cancer (CASPIAN): updated results from a randomised, controlled, open-label, phase 3 trial. Lancet Oncol 2021;22:51-65.

15. Horn L, Mansfield AS, Szczęsna A, et al. First-Line Atezolizumab plus Chemotherapy in Extensive-Stage Small-Cell Lung Cancer. N Engl J Med 2018;379:2220-9.

16. Ramalingam SS, Vansteenkiste J, Planchard D, et al. Overall Survival with Osimertinib in Untreated, EGFR-Mutated Advanced NSCLC. N Engl J Med 2020;382:41-50.

17. Puri S, Naqash AR, Elliott A, et al. Real-world multiomic characterization of small cell lung cancer subtypes to reveal differential expression of clinically relevant biomarkers. Wolters Kluwer Health; 2021.

18. Ito T, Matsubara D, Tanaka I, et al. Loss of YAP1 defines neuroendocrine differentiation of lung tumors. Cancer Sci 2016;107:1527-38. 
Table S1 Detailed pathological data of included patients

\begin{tabular}{|c|c|c|c|c|c|c|c|c|c|c|c|c|c|c|c|c|c|c|c|c|}
\hline $\begin{array}{l}\text { Patient } \\
\text { no. }\end{array}$ & Enroll & EGFR & $\begin{array}{l}\text { Histology } \\
\text { (pre) }\end{array}$ & $\begin{array}{l}\text { ASCL1 } \\
\text { H-score } \\
\text { (pre) }\end{array}$ & $\begin{array}{l}\text { NEUROD1 } \\
\text { H-score } \\
\text { (pre) }\end{array}$ & $\begin{array}{l}\text { POU2F3 } \\
\text { H-score } \\
\text { (pre) }\end{array}$ & $\begin{array}{l}\text { YAP1 } \\
\text { H-score } \\
\text { (pre) }\end{array}$ & $\begin{array}{l}\text { RB1 } \\
\text { (pre) }\end{array}$ & p53 (pre) & $\begin{array}{l}\text { Histology } \\
\text { (post) }\end{array}$ & $\begin{array}{l}\text { Combined } \\
\text { NSCLC } \\
\text { component }\end{array}$ & $\begin{array}{l}\text { ASCL1 } \\
\text { H-score } \\
\text { (post) }\end{array}$ & $\begin{array}{l}\text { NEUROD1 } \\
\text { H-score (post) }\end{array}$ & $\begin{array}{l}\text { POU2F3 } \\
\text { H-score } \\
\text { (post) }\end{array}$ & $\begin{array}{l}\text { YAP1 } \\
\text { H-score } \\
\text { (post) }\end{array}$ & $\begin{array}{l}\text { RB1 } \\
\text { (post) }\end{array}$ & p53 (post) & SUBTYPE (post) & $\begin{array}{l}\text { TTF-1 } \\
\text { (post) }\end{array}$ & $\begin{array}{l}\text { Large cell } \\
\text { feature in } \\
\text { SCLC }\end{array}$ \\
\hline 10 & Included & E19del & ADC & 0 & 0 & 0 & 120 & Loss & $\begin{array}{l}\text { Overexpression } \\
(80 \%)\end{array}$ & SCLC & - & 120 & 6 & 10 & 25 & Loss & $\begin{array}{l}\text { Overexpression } \\
(>90 \%)\end{array}$ & A & positive & present \\
\hline 14 & Included & E19del & $A D C$ & 10 & 0 & 10 & 15 & Loss & $\begin{array}{l}\text { Overexpression } \\
(>90 \%)\end{array}$ & SCLC & - & 140 & 0 & 0 & 10 & Loss & $\begin{array}{l}\text { Overexpression } \\
(80 \%)\end{array}$ & A & positive & absent \\
\hline 15 & Included & E19del & ADSCC & 10 & 0 & 10 & 220 & Loss & $\begin{array}{l}\text { Complete } \\
\text { absence (0\%) }\end{array}$ & SCLC & - & 240 & 30 & 0 & 5 & Loss & $\begin{array}{l}\text { Complete } \\
\text { absence (0\%) }\end{array}$ & A & positive & present \\
\hline 26 & Included & E19del & $A D C$ & N/A & N/A & $\mathrm{N} / \mathrm{A}$ & $\mathrm{N} / \mathrm{A}$ & N/A & $\mathrm{N} / \mathrm{A}$ & SCLC & - & 270 & 0 & 30 & 2 & Loss & $\begin{array}{l}\text { Overexpression } \\
(80 \%)\end{array}$ & A & positive & present \\
\hline 1 & Included & L858R & $A D C$ & 0 & 0 & 0 & 170 & Loss & $\begin{array}{l}\text { Overexpression } \\
(>90 \%)\end{array}$ & SCLC & - & 0 & 300 & 0 & 0 & Loss & $\begin{array}{l}\text { Overexpression } \\
(>90 \%)\end{array}$ & $\mathrm{N}$ & positive & absent \\
\hline 3 & Included & E19del & ADC & N/A & N/A & $\mathrm{N} / \mathrm{A}$ & N/A & N/A & $\mathrm{N} / \mathrm{A}$ & SCLC & - & 10 & 300 & 0 & 0 & Loss & Wild type (20\%) & $\mathrm{N}$ & positive & absent \\
\hline 4 & Included & E19del & $A D C$ & $N / A$ & N/A & $\mathrm{N} / \mathrm{A}$ & $\mathrm{N} / \mathrm{A}$ & N/A & $\mathrm{N} / \mathrm{A}$ & SCLC & - & 5 & 270 & 0 & 0 & Loss & $\begin{array}{l}\text { Overexpression } \\
(>90 \%)\end{array}$ & N & positive & absent \\
\hline 11 & Included & E19del & ADC & N/A & N/A & $\mathrm{N} / \mathrm{A}$ & N/A & N/A & $\mathrm{N} / \mathrm{A}$ & SCLC & - & 30 & 300 & 0 & 0 & $\begin{array}{l}\text { Intact } \\
(100 \%)\end{array}$ & $\begin{array}{l}\text { Overexpression } \\
(80 \%)\end{array}$ & $\mathrm{N}$ & positive & absent \\
\hline 12 & Included & L858R & ADC & N/A & N/A & $\mathrm{N} / \mathrm{A}$ & N/A & N/A & N/A & SCLC & - & 30 & 80 & 0 & 0 & $\begin{array}{l}\text { Intact } \\
(100 \%)\end{array}$ & $\begin{array}{l}\text { Overexpression } \\
(80 \%)\end{array}$ & $\mathrm{N}$ & positive & present \\
\hline 16 & Included & E19del & ADC & N/A & N/A & $\mathrm{N} / \mathrm{A}$ & N/A & N/A & $\mathrm{N} / \mathrm{A}$ & SCLC & - & 50 & 260 & 0 & 0 & Loss & N/A & $\mathrm{N}$ & positive & absent \\
\hline 20 & Included & E19del & ADC & 0 & 0 & 0 & 180 & Loss & $\begin{array}{l}\text { Complete } \\
\text { absence (0\%) }\end{array}$ & SCLC & - & 80 & 180 & 0 & 0 & Loss & $\begin{array}{l}\text { Complete } \\
\text { absence (0\%) }\end{array}$ & $\mathrm{N}$ & positive & absent \\
\hline 23 & Included & L858R & $\begin{array}{l}\mathrm{ADC} / \\
\mathrm{SCLC}^{\star}\end{array}$ & 0 & $120^{* *}$ & 0 & 10 & Loss & $\begin{array}{l}\text { Overexpression } \\
(>90 \%)\end{array}$ & SCLC & ADC & 0 & 240 & 0 & 0 & Loss & $\begin{array}{l}\text { Overexpression } \\
(>90 \%)\end{array}$ & $\mathrm{N}$ & positive & absent \\
\hline 9 & Included & L858R & ADC & N/A & N/A & $\mathrm{N} / \mathrm{A}$ & N/A & N/A & $\mathrm{N} / \mathrm{A}$ & SCLC & - & 0 & 0 & 5 & 0 & $\begin{array}{l}\text { Intact } \\
(50 \%)\end{array}$ & $\begin{array}{l}\text { Complete } \\
\text { absence (0\%) }\end{array}$ & TN & negative & absent \\
\hline 18 & Included & E19del & $A D C$ & N/A & N/A & $\mathrm{N} / \mathrm{A}$ & N/A & N/A & $\mathrm{N} / \mathrm{A}$ & SCLC & - & 5 & 0 & 40 & 30 & Loss & $\begin{array}{l}\text { Overexpression } \\
(>90 \%)\end{array}$ & TN & positive & present \\
\hline 22 & Included & E19del & ADC & N/A & N/A & $\mathrm{N} / \mathrm{A}$ & N/A & N/A & $\mathrm{N} / \mathrm{A}$ & SCLC & - & 0 & 0 & 0 & 0 & Loss & N/A & TN & positive & absent \\
\hline 27 & Included & E19del & ADC & N/A & N/A & $\mathrm{N} / \mathrm{A}$ & N/A & N/A & $\mathrm{N} / \mathrm{A}$ & SCLC & - & 0 & 0 & 0 & 0 & N/A & $\begin{array}{l}\text { Complete } \\
\text { absence (0\%) }\end{array}$ & TN & negative & absent \\
\hline 34 & Included & Leu858 & ADC & $\mathrm{N} / \mathrm{A}$ & N/A & $\mathrm{N} / \mathrm{A}$ & N/A & N/A & $\mathrm{N} / \mathrm{A}$ & SCLC & LCNEC & 5 & 0 & 30 & 65 & Loss & $\begin{array}{l}\text { Complete } \\
\text { absence (0\%) }\end{array}$ & TN & positive & present \\
\hline 2 & $\begin{array}{l}\text { Exploratory } \\
\text { Analysis }\end{array}$ & L858R & & & & & & & & SCLC & ADC & 140 & 0 & 0 & 7 & $\begin{array}{l}\text { Intact } \\
(50 \%)\end{array}$ & $\begin{array}{l}\text { Overexpression } \\
(80 \%)\end{array}$ & A & positive & present \\
\hline 37 & $\begin{array}{l}\text { Exploratory } \\
\text { Analysis }\end{array}$ & E19del/T & $790 \mathrm{M}$ & & & & & & & SCLC & $\begin{array}{l}\text { ADC/ } \\
\text { LCNEC }\end{array}$ & 120 & 0 & 0 & 0 & $\begin{array}{l}\text { Intact } \\
(100 \%)\end{array}$ & $\begin{array}{l}\text { Complete } \\
\text { absence (0\%) }\end{array}$ & A & positive & present \\
\hline 25 & $\begin{array}{l}\text { Exploratory } \\
\text { Analysis }\end{array}$ & L858R & & & & & & & & SCLC & ADC & 0 & 250 & 0 & 1 & Loss & $\begin{array}{l}\text { Overexpression } \\
(80 \%)\end{array}$ & $\mathrm{N}$ & positive & absent \\
\hline 30 & $\begin{array}{l}\text { Exploratory } \\
\text { Analysis }\end{array}$ & E19del & & & & & & & & SCLC & ADC & 0 & 220 & 10 & 5 & $\begin{array}{l}\text { Intact } \\
(20 \%)\end{array}$ & $\begin{array}{l}\text { Overexpression } \\
(70 \%)\end{array}$ & $\mathrm{N}$ & positive & absent \\
\hline 40 & $\begin{array}{l}\text { Exploratory } \\
\text { Analysis }\end{array}$ & E19del & & & & & & & & SCLC & ADC & 5 & 300 & 10 & 0 & Loss & Wild type $(30 \%)$ & $\mathrm{N}$ & positive & present \\
\hline 29 & $\begin{array}{l}\text { Exploratory } \\
\text { Analysis }\end{array}$ & E19del & & & & & & & & SCLC & LCNEC & 30 & 3 & 50 & 0 & Loss & $\begin{array}{l}\text { Complete } \\
\text { absence (0\%) }\end{array}$ & TN & positive & present \\
\hline
\end{tabular}




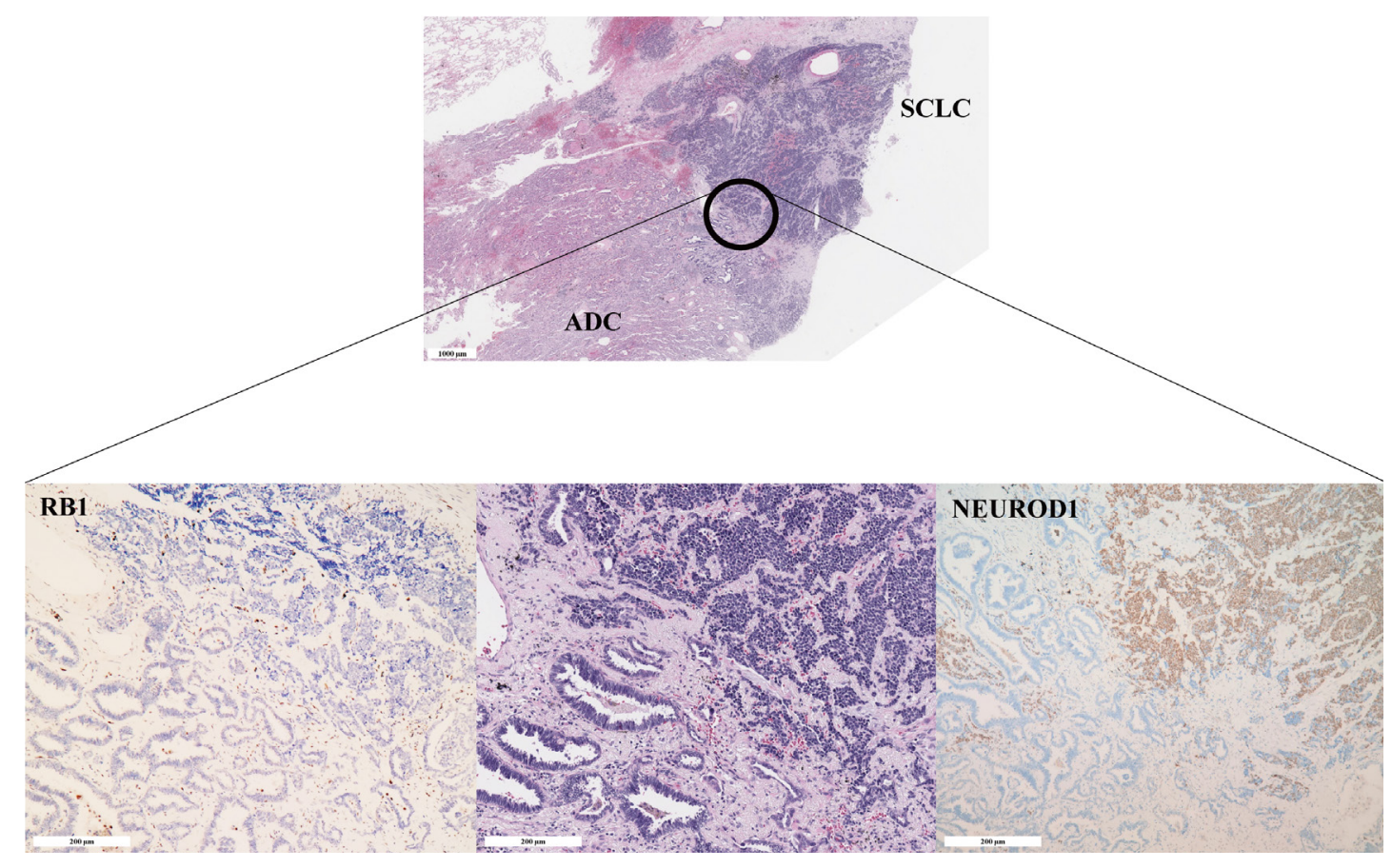

Figure S1 Representative images for de novo combined SCLC showing the spatial difference in NEUROD1 and RB1 staining (hematoxylineosin, RB1 and NEUROD1 stain; top scale bars $=1,000 \mu \mathrm{m}$, bottom scale bars $=200 \mu \mathrm{m})$. SCLC, small cell lung cancer. 